\title{
The potential role of SPECT/CT in the clinical management of COVID-19 lung cancer patients undergoing radiotherapy
}

\author{
Paolo Borghetti ${ }^{1}$. Cristian Toraci ${ }^{2}$ Jessica Imbrescia ${ }^{1}$. Giulia Volpi ${ }^{1}$ Silvia Lucchini ${ }^{3}$. Andrea Emanuele Guerini ${ }^{1}$. \\ Alessandro Magli $^{4} \cdot$ Luigi Spiazzi $^{2}$ - Alfonso Reginelli ${ }^{5}$. Salvatore Cappabianca ${ }^{5}$. Stefano Maria Magrini ${ }^{1}$. \\ Valerio Nardone ${ }^{5}$ (i)
}

Received: 14 July 2021 / Accepted: 26 July 2021 / Published online: 28 July 2021

(C) The Japanese Society of Nuclear Medicine 2021

\section{Introduction}

Dear Sir, we read with great interest the paper of Ozturk et al. [1], investigating the clinical benefit of Q-SPECT/CT in diagnosing perfusion defects and the tendency to thrombosis in COVID-19 patients.

At this regard, we would like to suggest the potential role of this examination in the clinical management of lung cancer patients with a previous pneumonitis of COVID-19.

Early reports of late effects of pandemic on pulmonary functions are worrisome [2], and especially lung fibrosis could be a serious concern for lung cancer patients who need to undergo radiotherapy, thus the assessment of the lung functionality before RT is pivotal.

In this regard, SPECT/CT is able to provide a quantitative assessment of the function in vascular/alveolar units [3], which can be used in RT planning to reduce the risk of radiation-induced toxicity [4].

We performed a SPECT/CT examination in a locally advanced lung cancer patient with a previous COVID-19 pneumonitis, to understand the correlation between $\mathrm{CT}$ and SPECT/CT imaging and to optimize RT plan, taking in consideration the lung functionality.

Valerio Nardone

v.nardone@hotmail.it

1 Radiation Oncology Department, University and Spedali Civili of Brescia, Brescia, Italy

2 Medical Physics Department, Spedali Civili of Brescia, Brescia, Italy

3 Nuclear Medicine Department, Spedali Civili of Brescia, Brescia, Italy

4 Department of Radiation Oncology, Udine General Hospital, Udine, Italy

5 Department of Precision Medicine, University of Campania "L. Vanvitelli", Naples, Italy
SPECT/CT examination showed hypoperfusion at known lung neoplastic lesion and a globally nonhomogeneous perfusion in the remaining segments. No pronounced areas of hypofixation were described.

Volumes of initial pneumonia were $411 \mathrm{~cm}^{3}$ and $413 \mathrm{~cm}^{3}$ on the right and left lung, respectively. Volumes of residual interstitial pneumonia at the restaging CT before CCRT were $13.3 \mathrm{~cm}^{3}$ and $10.4 \mathrm{~cm}^{3}$ on the right and left lung, respectively (see Fig. 1). Despite the clear reduction at standard $\mathrm{CT}$, the co-registration of these areas with SPECT/CT demonstrated that the areas of previous COVID pneumonia showed a remarkable residual hypoperfusion.

Presumably, the changes of lung parenchyma such as the fibrosis are unlikely to regress in patients with severe COVID-19 who survive, although long-term functional impairment is still unknown [2].

With these premises, in the next years, an increasing number of the patients diagnosed with lung cancer could wear the lung scars of a previous pandemic infection.

When these patients will be referred for radiotherapy, the poor lung functionality could jeopardize the achievement of a curative approach.

Mean lung dose (MLD) and volume of lung receiving 20 Gy (V20) represent the most commonly used lung dose constraints in clinical practice. It is important to underline that there is no absolute cut-off of these dose constraints below which there is no risk for radiation pneumonitis, despite that it is generally recommended to limit MLD to $<20 / 23$ Gy and $\mathrm{V} 20<30-35 \%$ [5].

$\mathrm{SPECT} / \mathrm{CT}$ is able to provide information on the distribution of pulmonary blood flow, where perfused areas equate with normal functioning lung [3]. These spatial information can be used to calculate functional dose constraints (fDC), with different techniques.

It is noteworthy to underline that despite the literature evidence, SPECT/CT examinations are not performed 

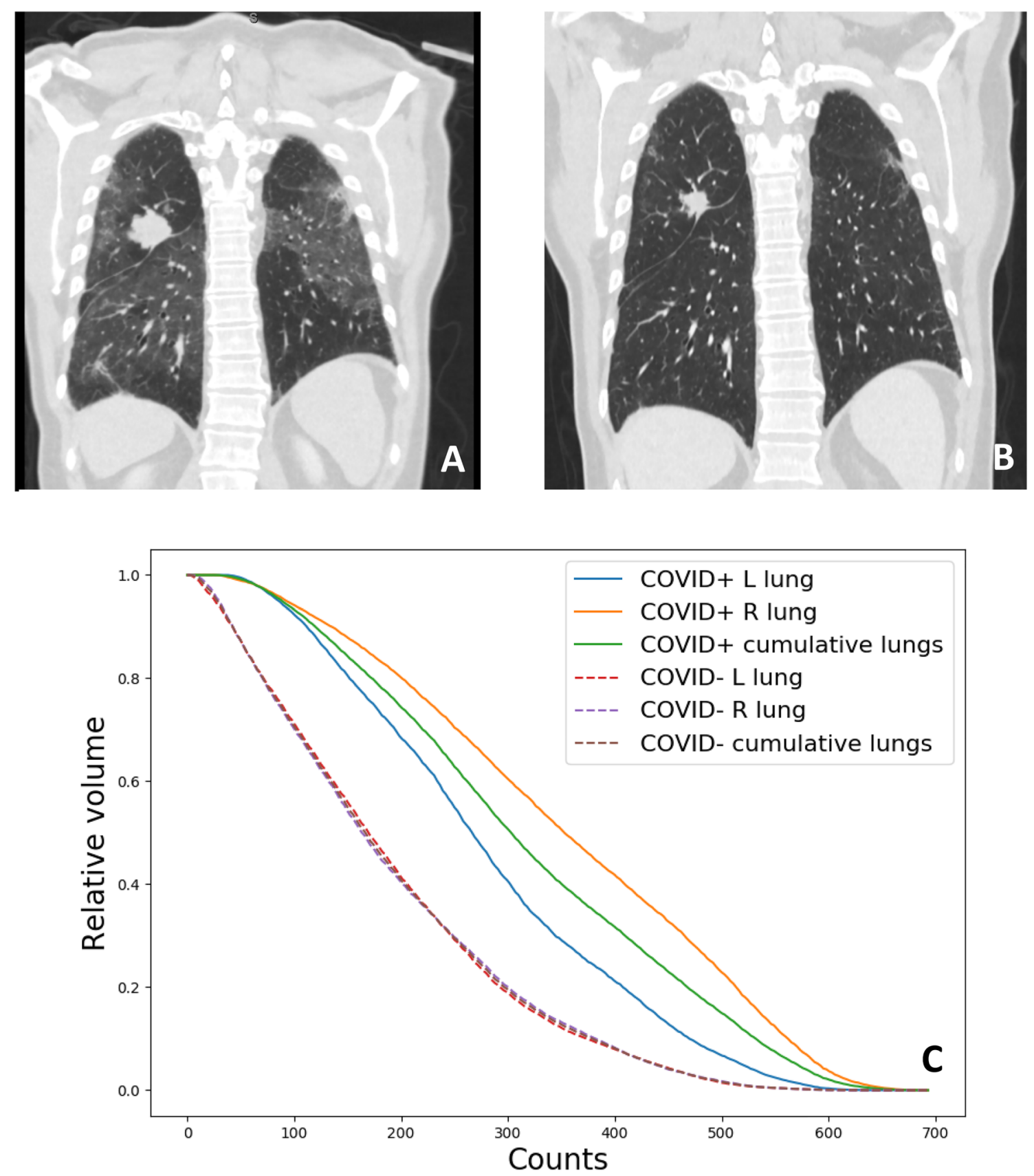

Fig. 1 A Basal CT-scan showing signs of bilateral interstitial pneumonia. B Pre-treatment CT, showing almost complete resolution of COVID pneumonia. $\mathbf{C}$ The data of perfusion was measured in terms of detected photons: higher values are generated by high numbers of

routinely in the clinical management of lung cancer patients undergoing RT.

Conversely, in the case reported, we have shown the potential of the quantitative assessment of the lung function with SPECT/CT in the RT planning of a COVID patient.

Concluding, SPECT/CT has already proved to be useful to predict RT-induced lung toxicity, so that in the next future photons detected, therefore stating a lower and slow perfusion. The co-registration showed a clear tracer hypofixation in areas with pneumonia compared to those not affected

this examination could be essential in patients with a previous COVID infection and a poor lung functionality. 


\section{Declarations}

Conflict of interest All the authors report no conflict of interest concerning the materials or method used in this study or the findings specified in this paper.

\section{References}

1. Ozturk BC, Atahan E. Investigation of perfusion defects by Q-SPECT/CT in patients with mild-to-moderate course of COVID-19 and low clinical probability for pulmonary embolism. Ann Nucl Med. 2021. https://doi.org/10.1007/ s12149-021-01647-y.

2. Spagnolo P, Balestro E, Aliberti S, Cocconcelli E, Biondini D, Casa GD, Sverzellati N, Maher TM. Pulmonary fibrosis secondary to COVID-19: a call to arms? Lancet Respir Med. 2020;8(8):7502. https://doi.org/10.1016/S2213-2600(20)30222-8.

3. Boersma LJ, Damen EMF, de Boer RW, Muller SH, Roos CM, Valdés Olmos RA, van Zandwijk N, Lebesque JV. Dose-effect relations for local functional and structural changes of the lung after irradiation for malignant lymphoma. Radiother Oncol. 1994;32(3):201-9. https://doi.org/10.1016/0167-8140(94) 90019-1.

4. Farr KP, Kallehauge JF, Møller DS, Khalil AA, Kramer S, Bluhme $\mathrm{H}$, Morsing A, Grau C. Inclusion of functional information from perfusion SPECT improves predictive value of dose-volume parameters in lung toxicity outcome after radiotherapy for nonsmall cell lung cancer: a prospective study. Radiother Oncol. 2015;117(1):9-16. https://doi.org/10.1016/j.radonc.2015.08.005.

5. Marks LB, Bentzen SM, Deasy JO, Kong F-MS, Bradley JD, Vogelius IS, El Naqa I, Hubbs JL, Lebesque JV, Timmerman RD, Martel MK, Jackson A. Radiation dose-volume effects in the lung. Int J Radiat Oncol Biol Phys. 2010;76(3 Suppl):S70-6. https://doi. org/10.1016/j.ijrobp.2009.06.091.

Publisher's Note Springer Nature remains neutral with regard to jurisdictional claims in published maps and institutional affiliations. 